\title{
The azido ligand: a useful tool in designing chain compounds exhibiting alternating ferro- and antiferro-magnetic interactions
}

\author{
Guillaume Viau, ${ }^{a}$ Maria Grazia Lombardi, ${ }^{b}$ Giovanni De Munno, ${ }^{* b}$ Miguel Julve, ${ }^{* a}$ Francesc Lloret, ${ }^{a}$ Juan $^{\circ}$ \\ Faus, ${ }^{a}$ Andrea Caneschi ${ }^{c}$ and Juan Modesto Clemente-Juan ${ }^{a}$ \\ a Departament de Química Inorganica, Facultat de Química de la Universitat de València, Dr. Moliner 50, 46100 Burjassot \\ (València), Spain \\ ${ }^{b}$ Dipartimento di Chimica, Università degli Studi della Calabria, 87030 Arcavacata di Rende, Cosenza, Italy \\ c Dipartimento di Chimica, Università degli Studi di Firenze, 75/77 Via Maragliano, 50144 Firenze, Italy
}

\begin{abstract}
A one-pot reaction of $\mathrm{NiII}^{\mathrm{II}}, \mathrm{Co}^{\mathrm{II}} 2, \mathrm{Fe}^{\mathrm{II}} 3$ and $\mathrm{Mn}^{\mathrm{II}} 4$ with $2,2^{\prime}$-bipyridine (bipy) and azide in water leads to $\left[\mathrm{M}(\text { bipy })\left(\mathrm{N}_{3}\right)_{2}\right]_{n}$ chains where the metal ion is alternatively bridged by double end-on (EO) and end-to-end (EE) azido bridges; theoretical analysis of the variable-temperature magnetic susceptibility data of 1 and 4 reveals the occurrence of intrachain alternating ferro- (through EO) and antiferro-magnetic (through $\mathrm{EE}$ ) interactions.
\end{abstract}

A safe strategy in designing alternating chains with ferro- and antiferro-magnetic interactions has been proposed recently, ${ }^{1}$ and the first honeycomb-layered materials of formula $\left[\mathrm{M}(\right.$ bipym $\left.)\left(\mathrm{N}_{3}\right)_{4}\right] \quad\left(\mathrm{M}=\mathrm{Mn}, \mathrm{Fe}, \mathrm{Co}\right.$; bipym $=2,2^{\prime}$-bipyrimidine) $)^{2}$ with alternating ferro- (through end-on azido, EO) and antiferro-magnetic (through bischelating bipym) interactions have also been reported. Replacement of bipym by bipy (2,2'-bipyridine) yields chain compounds of formula $\left[\mathrm{M}(\right.$ bipy $\left.)\left(\mathrm{N}_{3}\right)_{4}\right]\left(\mathrm{M}=\mathrm{NiII}\right.$ 1, $\mathrm{Co}^{\mathrm{II}}$ 2, $\mathrm{Fe}^{\mathrm{II}} 3, \mathrm{Mn}^{\mathrm{II}}$ 4) with alternating $\mathrm{EO}$ and end-to-end (EE) azido bridges and exhibiting ferro- (through EO) ${ }^{3}$ and antiferro-magnetic (EE) ${ }^{4}$ interactions. Here, we report the synthesis, crystal structure and preliminary magnetic study of compounds $\mathbf{1}-\mathbf{3}$. The magnetic properties of 4,5 which is isostructural with 2 and $\mathbf{3}$, were reinvestigated for the sake of comparison and the first theoretical analysis of the magnetic behaviour of $\mathbf{1}$ and $\mathbf{4}$ through a quantum spin model is also reported.

The one-dimensional compounds were obtained as polycrystalline green (1), orange (2) and red-brown (3) powders by adding an aqueous solution $\left(25 \mathrm{~cm}^{3}\right)$ of $\mathrm{NaN}_{3}[2 \mathrm{mmol}(\mathbf{1})$ or 10 mmol $(2,3)]$ to a methanolic solution $\left(5 \mathrm{~cm}^{3}\right)$ containing bipy $(1$ $\mathrm{mmol})$ and either $\mathrm{Ni}\left(\mathrm{NO}_{3}\right)_{2} \cdot 6 \mathrm{H}_{2} \mathrm{O}(1 \mathrm{mmol}, 1), \mathrm{Co}\left(\mathrm{NO}_{3}\right)_{2} \cdot 6 \mathrm{H}_{2} \mathrm{O}$ (1 mmol, 2) or $\mathrm{FeCl}_{2}(1 \mathrm{mmol}, 3)$. The solid samples were filtered off and thoroughly washed with methanol and diethyl ether (yield $c a .50 \%$ ). X-Ray quality polyhedral (1), arrow shaped (2) and prismatic (3) single crystals were grown by slow diffusion techniques in test $(\mathbf{2}, \mathbf{3})$ or $\mathrm{H}$-shaped $(\mathbf{1})$ tubes. $\dagger$

The structures of 1-4 are made up of alternating chains where the metal ions are alternately linked by two EO and two EE azido bridges (Fig. 1). $\neq$ Two nitrogen atoms of the bipy ligand complete a distorted $\mathrm{MN}_{6}$ octahedral environment around each metal atom. The EE and EO bridges are propagated within the chain with a mutual perpendicular arrangement. The value of the $\mathrm{M}(1)-\mathrm{N}(3)-\mathrm{M}(1 \mathrm{a})$ angle is 101.5(1) 1, 100.9(1) 2 and $100.8(1)^{\circ} \mathbf{3}$ in good agreement with the value of $101.0(2)^{\circ}$ observed in the parent compound of $\mathrm{Mn}^{\mathrm{II}} 4 .^{5}$ The EE bridges adopt a chair conformation for the $\mathrm{M}-\left(\mathrm{N}_{3}\right)_{2}-\mathrm{M}$ unit. The values of the $\mathrm{M}(1)-\mathrm{N}(6)-\mathrm{N}(7)$ and $\mathrm{N}(7)-\mathrm{N}(8)-\mathrm{M}(1 \mathrm{~b})$ angles are 118.2(2) and 129.9(3) $)^{\circ}$ in 1, 127.1(2) and 129.9(2) in $\mathbf{2}$ and 126.5(2) and $131.2(1)^{\circ}$ in 3 [131.1(5) and $127.3(5)^{\circ}$ in 4]. The dihedral angle between the $\mathrm{N}(6) \mathrm{N}(7) \mathrm{N}(8) \mathrm{N}(6 \mathrm{a}) \mathrm{N}(7 \mathrm{a}) \mathrm{N}(8 \mathrm{a})$ and $\mathrm{MN}(6) \mathrm{N}(8 \mathrm{~b})$ planes is 35.2(1) $\mathbf{1}, 24.9(1) 2$ and $23.7(1)^{\circ} \mathbf{3}\left(41.9^{\circ}\right.$ in 4). The intrachain metal-metal separation through $\mathrm{EO}$ azido is 3.248(1) 1, 3.322(1) 2 and 3.367(1) $\AA 3$ [3.455(6) $\AA$ in 4] and that through EE azido is 5.117(2) 1, 5.279(2) 2 and 5.317(2) $\AA$ 3 [5.343(5) $\AA$ in 4]. The shortest interchain metal-metal separation is $8 \AA$ [8.462(3) $\mathbf{1}, 8.115(4) 2$, 8.063(3) $\AA$ 3].

Magnetic susceptibility measurements (290-2 K) on polycrystalline samples of $\mathbf{4}$ (Fig. 2) are consistent with overall antiferromagnetic behaviour. Least-squares analysis of the $\chi_{\mathrm{M}} T$ data of $4 \S$ leads to $J_{1}=2.5 \mathrm{~cm}^{-1}, J_{2}=-6.3 \mathrm{~cm}^{-1}$ and $g=2.0$. $J_{1}$ and $J_{2}$ are associated with the exchange coupling through $\mathrm{EO}$ and EE azido bridges, respectively. The $\chi_{\mathrm{M}} T$ value of 1 (Fig. 3 ) smoothly increases as the temperature is lowered, attains a plateau at $70 \mathrm{~K}$ and quickly decreases in the lower temperature region. The susceptibility curve (inset of Fig. 3) increases monotonically when cooling, attains a shoulder in the temperature range $50-20 \mathrm{~K}$ and then increases achieving a maximum at $8.5 \mathrm{~K}$. The shape of the shoulder is field dependent at $T<50 \mathrm{~K}$ but the temperature of the maximum remains unchanged. The theoretical analysis of the magnetic data of $\mathbf{1}^{6}$ matches well the experimental data in the temperature range 290-55 K. The best fit parameters are $J_{1}$ (coupling through EO

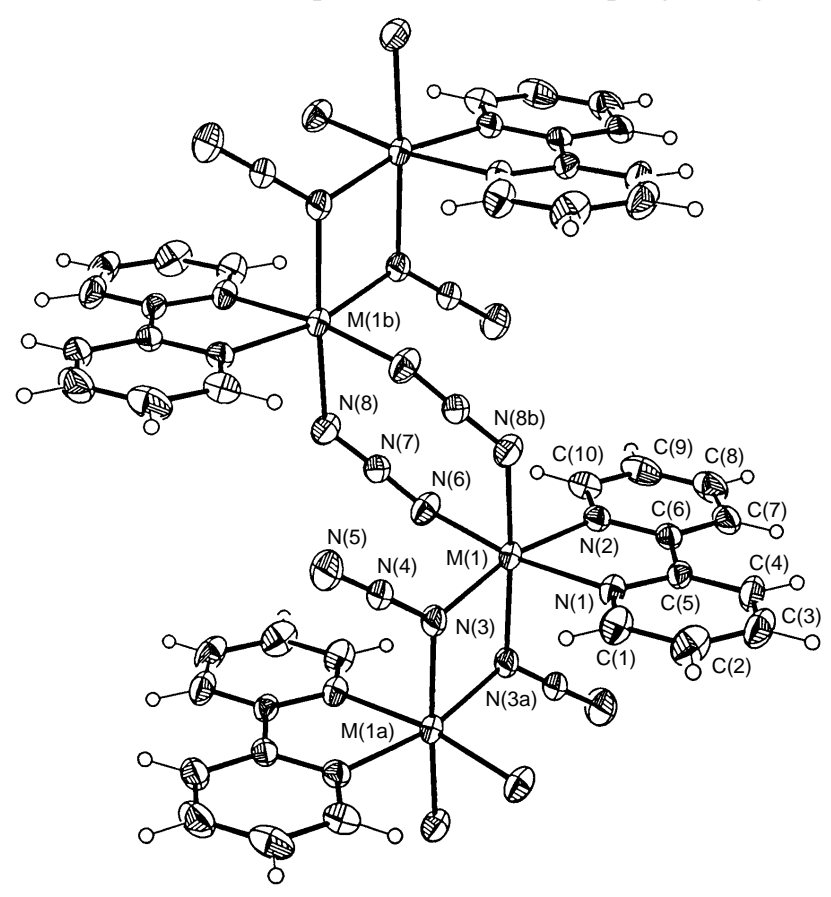

Fig. 1 Crystal structure of $\left[\mathrm{M}(\text { bipy })\left(\mathrm{N}_{3}\right)_{2}\right]_{n}(\mathrm{M}=\mathrm{Ni}$ 1, Fe 2, Co 3) showing the atom numbering (ellipsoids are at the $30 \%$ probability level). The M-N bond lengths vary in the ranges 2.064(3)-2.145(4) $\AA$ for $\mathbf{1}$, $2.118(2)-2.189$ (2) A for 2 and 2.132(2)-2.240(2) $\AA$ for 3 . The values of the $\mathrm{M}(1)-\mathrm{N}(3)-\mathrm{M}(1 \mathrm{a})$ bond angles are 101.5(1) (1), 100.9(1) (2) and $100.8(1)^{\circ}$ (3). The EO and EE azide groups are quasilinear [the smallest N(3)-N(4)$\mathrm{N}(5)$ and $\mathrm{N}(6)-\mathrm{N}(7)-\mathrm{N}(8)$ bond angles are 178.1(3) and $176.7(2)^{\circ}$, respectively]. (Symmetry code for 1 : a $-x, 1-y,-z$; b $1-x, 1-y,-z$. Symmetry code for 2 and 3: a $-x, 1-y, 1-z$; b $1-x, 1-y, 1-z$.) 


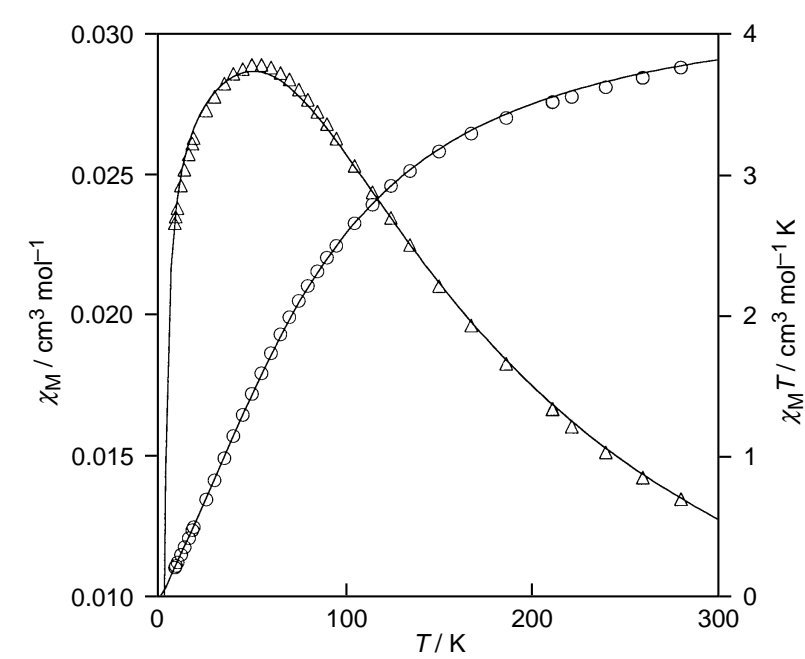

Fig. $2 \chi_{M} T(\bigcirc)$ and $\chi_{M}(\triangle)$ vs. $T$ plots for complex 4. The solid line corresponds to the fit to a model outlined in ref. 6 .

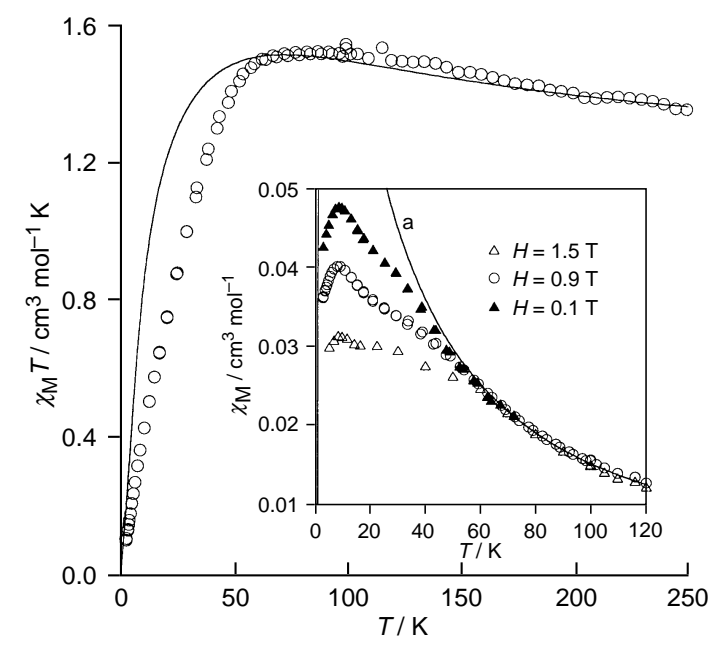

Fig. $3 \chi_{\mathrm{M}} T v s$. $T$ plot for complex 1 . The inset shows the field dependence of the susceptibility in the low-temperature region. The solid line corresponds to the fit to a model outlined in ref. 6.

azido) $=26 \mathrm{~cm}^{-1}, J_{2}$ (coupling through EE azido) $=-2.6$ $\mathrm{cm}^{-1}$ and $g=2.17$. The experimental data are smaller than the calculated ones at $T<55 \mathrm{~K}$ due to the occurrence of the mentioned shoulder precluding thus an accurate determination of the values of the exchange coupling parameters. The magnetic behaviour of $2 \mathbb{T}$ is exotic too: $\chi_{\mathrm{M}} T$ decreases when cooling down until reaching a minimum at $29 \mathrm{~K}$, then increases to attain a sharp maximum at $12 \mathrm{~K}$ and finally decreases very quickly in the lower temperature region. As in $\mathbf{1}$, the susceptibility curve also shows a shoulder but at higher temperatures $(50-100 \mathrm{~K})$, exhibiting a maximum at $8.5 \mathrm{~K}$ under an applied field $H$ of 0.1 T. However, this maximum disappears for $H>0.5 \mathrm{~T}$. Given that all the cobalt atoms in 2 are equivalent, the occurrence of a minimum in the $\chi_{\mathrm{M}} T$ curve cannot be attributed to ferrimagnetism arising from a noncompensation of the local $g$-factors but to a intrachain spin canting. An interchain antiferromagnetic coupling would account for the observed maximum of susceptibility at $H<0.5 \mathrm{~T}$. Stronger fields overcome this antiferromagnetic coupling leading to the observed metamagnetic-like behaviour of $\mathbf{2}$. Similar behaviour was observed for 3 . This peculiar behaviour of $\mathbf{1 - 3}$ is a fascinating feature that could be attributed to the occurrence of competing ferro- and antiferro-magnetic interactions together with a relevant local anisotropy. In the lack of a significant anisotropy, this feature is not observed (in the case of $\mathbf{4}$ and alternating $\mathrm{Cu}^{\mathrm{II}}$ chains). ${ }^{1}$ Peculiar susceptibility and magnetization curves were also observed for honeycomb- layered materials with alternating intralayer ferro- and antiferro-magnetic interactions. ${ }^{2}$ It is interesting to note that the spin vectors in the ferromagnetic chains are aligned parallel to the chain axis whereas in the case of the antiferromagnetic (or ferrimagnetic) chains they are arranged perpendicular to the chain axis in order to reconciliate both the dipolar and exchange interactions. ${ }^{7}$ The question at hand concerning the chains exhibiting alternating ferro- and antiferro-magnetic interactions is to know the arrangement of the spin vectors for them. Our feeling is that the peculiar magnetic behaviour of 1-3 is related to the competition among the local anisotropy and the dipolar and exchange interactions.

This work was supported by the Spanish DGICYT (Projects PB94-1002 and PB94-0998) and the Italian Ministero dell'Università e della Ricerca Scientifica e Tecnologica.

\section{Footnotes}

* E-mail: miguel.julve@uv.es

$\dagger$ Satisfactory elemental analyses $(\mathrm{C}, \mathrm{H}, \mathrm{N})$ were obtained.

$\ddagger X$-Ray crystal structure analyses: Siemens $\mathrm{R} 3 \mathrm{~m} / \mathrm{V}$ automatic diffractometer, Mo-K $\alpha$ radiation, $\lambda=0.71073 \AA$, graphite-monochromator, 295 K. Lorentz polarization and absorption corrections. Data collection, solution and refinement: $\omega-2 \theta$, standard Patterson methods and subsequent Fourier recycling, SHELX-PLUS computer program. Compounds $\mathbf{1}-\mathbf{3}$ have the same formula, $\mathrm{C}_{10} \mathrm{H}_{8} \mathrm{MN}_{8}(\mathrm{M}=\mathrm{Ni} \mathbf{1}, \mathrm{Co} 2, \mathrm{Fe} 3)$, triclinic, space group $P \overline{1}$ ( 2 and $\mathbf{3}$ are isostructural). For $\mathbf{1}, a=7.017(2), b=9.662(2)$, $c=10.279(3) \AA, \alpha=111.12(2), \beta=99.56(2), \gamma=107.34(2)^{\circ}$, $U=590.6(3) \AA^{3}, Z=2, D_{\mathrm{c}}=1.681 \mathrm{~g} \mathrm{~cm}^{-3}$, crystal size $0.21 \times 0.14 \times$ $0.05 \mathrm{~mm} .2869$ reflections measured in the range $3<2 \theta<54^{\circ}, 2586$ unique and 1952 assumed as observed with $I>3 \sigma(I) ; R=0.035$ and $R_{\mathrm{w}}=0.036$. For $2, a=7.311(2), b=9.243(3), c=10.061(4) \AA$, $\alpha=110.93(3), \beta=102.61(3), \gamma=102.93(3)^{\circ}, U=584.8(3) \AA^{3}, Z=2$, $D_{\mathrm{c}}=1.699 \mathrm{~g} \mathrm{~cm}^{-3}$, crystal size $0.40 \times 0.28 \times 0.24 \mathrm{~mm} .2843$ reflections measured in the range $3<2 \theta<54^{\circ}, 2589$ unique and 2288 assumed as observed with $I>3 \sigma(I) ; R=0.029$ and $R_{\mathrm{w}}=0.036$. For 3, $a=7.448(2)$, $b=9.195(3), c=10.020(3) \AA, \alpha=111.00(2), \beta=104.07(2)$, $\gamma=101.53(2)^{\circ}, U=589.4(3) \AA^{3}, Z=2, D_{\mathrm{c}}=1.668 \mathrm{~g} \mathrm{~cm}^{-3}$, crystal size $0.50 \times 0.21 \times 0.29 \mathrm{~mm} .2856$ reflections measured in the range $3<2 \theta<$ $54^{\circ}, 2589$ unique and 2277 assumed as observed with $I>3 \sigma(I) ; R=0.027$ and $R_{\mathrm{w}}=0.031$. Atomic coordinates, bond lengths and angles, and thermal parameters have been deposited at the Cambridge Crystallographic Data Centre (CCDC). See Information for Authors, Issue 1. Any request to the CCDC for this material should quote the full literature citation and the reference number 182/468.

$\S$ The numerical expressions used for the analysis of the magnetic properties of 1 and 4 were obtained using the isotropic Hamiltonian $\hat{H}=-J \Sigma$ $\left[\hat{S}_{2 i} \hat{S}_{2 i-1}-\alpha \hat{S}_{2 i} \hat{S}_{2 i+1}\right]$ by fitting the theoretical susceptibility curves of the rings up to ten $S=1$ (1) or six $S=5 / 2$ (2) local spins instead of open chains. The theoretical development has been elaborated in ref. 6 .

II Magnetic data for complex $\mathbf{2}$ are available from the authors.

\section{References}

1 G. De Munno, M. Julve, F. Lloret, J. Faus, M. Verdaguer and A. Caneschi, Angew. Chem., Int. Ed. Engl., 1993, 32, 1046; Inorg. Chem., 1995, 34, 157.

2 G. De Munno, M. Julve, G. Viau, F. Lloret, J. Faus and D. Viterbo, Angew. Chem., Int. Ed. Engl., 1996, 35, 1807; R. Cortés, L. Lezama, J. L. Pizarro, M. I. Arriortua and T. Fojo, Angew. Chem., Int. Ed. Engl., 1996, 35, 1810; G. De Munno, T. Poerio, G. Viau, M. Julve, F. Lloret, Y. Journaux and E. Rivière, Chem. Commun., 1996, 2587.

3 R. Cortés, J. L. Pizarro, L. Lezama, M. I. Arriortua and T. Rojo, Inorg. Chem., 1994, 33, 2697; A. Escuer, R. Vicente, J. Ribas and X. Solans, Inorg. Chem., 1995, 34, 1793; M. G. Drew, C. J. Harding and J. Nelson, Inorg. Chim. Acta, 1996, 246, 73.

4 O. Kahn, Comments Inorg. Chem., 1984, 3, 105; J. Ribas, M. Monfort, C. Diaz, C. Bastos and X. Solans, Inorg. Chem., 1993, 32, 3557.

5 R. Cortés, L. Lezama, J. L. Pizzaro, M. I. Arriortua, X. Solans and T. Rojo, Angew. Chem., Int. Ed. Engl., 1994, 33, 2488; R. Cortés, M. Drillon, X. Solans, L. Lezama and T. Rojo, Inorg. Chem., 1997, 36, 677.

6 J. J. Borrás-Almenar, J. M. Clemente, E. Coronado and F. Lloret, Chem. Phys. Lett., in press.

7 D. Gatteschi, O. Guillou, C. Zanchini, R. Sessoli, O. Kahn, M. Verdaguer and Y. Pei, Inorg. Chem., 1989, 28, 287.

Received in Basel, Switzerland, 21st February 1997; Com. $7 / 01219 E$ 